Conference Article

\title{
The effect of micro-swinging on joint formation in linear friction welding
}

\author{
Wenya $\mathrm{Li}^{1, *}$, Jia Guo ${ }^{1}$, Xiawei Yang ${ }^{1}$, Tiejun $\mathrm{Ma}^{1}$ and Achilleas Vairis ${ }^{2}$ \\ ${ }^{1}$ State Key Laboratory of Solidification Processing, Shaanxi Key Laboratory of Friction Welding Technologies, \\ Northwestern Polytechnical University, Xi'an 710072, Shaanxi, PR China \\ ${ }^{2}$ Mechanical Engineering Department, TEI of Crete, Heraklion, Crete 71004, Greece
}

Received 2 September 2014; Accepted 22 September 2014

\begin{abstract}
A 3D Eulerian numerical model was developed to investigate the effect of micro-swing on joint formation during workpiece oscillation in linear friction welding (LFW). The temperature field and axial shortening history for different amplitudes of micro-swing have been studied. Results show that the amplitude of micro-swing influences flash morphology and axial shortening. The micro-swing contributes to the extrusion of viscoplastic metal and the formation of flash during the LFW process. Flash volume and axial shortening increase as the amplitude of micro-swing becomes larger. When the amplitude of micro-swing is more than a critical value, a sudden change of axial shortening (different from the period of the oscillation cycle) would occur at certain welding time, which change would also have a periodic nature. Although different amplitudes of micro-swing affect joint morphology, the inner temperature field and the highest temperature of joints remain constant. The high temperature region inside the joints remains about the same, when the amplitude of micro-swing is constant. This indicates that the heat generated through plastic deformation could maintain the welding process, and so that the welding process would enter the equilibrium phase.
\end{abstract}

Keywords Linear Friction Welding, Micro-swing, Axial shortening, Numerical simulation

\section{Introduction}

Linear Friction welding (LFW) is a solid-state process for joining materials together through intimate contact of a plasticised interface, which is generated by frictional heat produced as one component is moved under pressure in a direct reciprocating mode relative to another. Compared to traditional rotary friction welding (RFW) [1] and innovative friction stir welding (FSW) [2-5], LFW is mainly used to weld non-axisymmetric components. LFW has been used to weld titanium alloys, nickel-based superalloys, steels, Al alloys, and dissimilar alloys [6]. As a key manufacturing technology, LFW is being used to produce aeroengine blisks (bladed integrated disk) [7].

According to the analysis of the microstructure evolution of LFW joints [8-10], LFW has four distinct phases which include the initial phase, transition phase, equilibrium phase and deceleration (or forging) phase. The initial phase and transition phase last normally less than $1 \mathrm{~s}$. Their impact on the welding process is usually ignored by researchers. During a recent LFW experiment, however, it was identified through high-speed photography that the oscillatoring specimen does not move rigidly in the oscillation direction, but has a micro-swing relative to the oscillation plane, as shown in Fig. 1.

In addition, LFW is a complicated and strongly thermomechanically coupled physical process. It is very difficult to

\footnotetext{
* E-mail address: liwy@nwpu.edu.cn

ISSN: 1791-2377 @ 2014 Kavala Institute of Technology. All rights reserved.
}

study its nature merely by welding experiments. Therefore, the finite element method is used to investigate the effect of micro-swing on the LFW process.

Up to now, different researchers [11-20] have built 2D or 3D thermo-mechanically coupled models in the Lagrangian frame using commercial software like DEFORM, ABAQUS, ANSYS or FORGE. The predicted temperature and axial shortening under certain welding conditions were comparable to experiments, but excessive element distortion and flash formation cannot be resolved effectively for a numerical solution. One measure to cope with excessive element distortion in ABAQUS is Arbitrary Lagrangian Eulerian (ALE) adaptive mesh controls [11], but the ability of this method to handle 3D large deformation problems is limited. Therefore, the Eulerian algorithm is commonly used in cases where extensive deformation has occurred, which divides the space into fixed meshes, and materials flow in the meshes. In this study, the Eulerian method is for the first time used to model the LFW process.

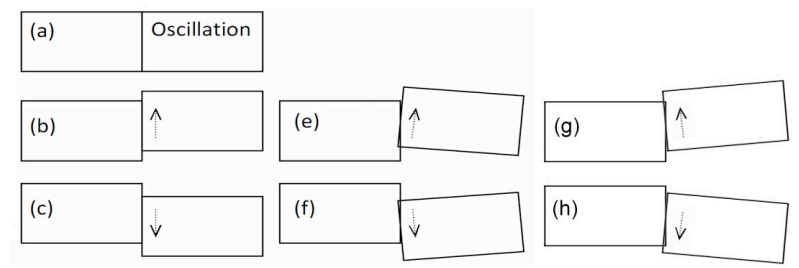

Fig. 1. Movement of the oscillating workpiece (a), ideally without (b,c) and in reality with (e-h) micro-swing. 


\section{Numerical analysis}

\subsection{Finite element model}

Considering the local large deformation during the LFW process, a 3D Eulerian model of LFW was developed using ABAQUS as shown in Fig. 2 (the surrounding void material has been hidden to visualize components). The workpiece was simplified as a single part, instead of two workpieces as has been used by Turner et al [21]. It had a length of $18 \mathrm{~mm}$, width of $10 \mathrm{~mm}$ and height of $60 \mathrm{~mm}$. The mesh size of workpiece was $0.8 \mathrm{~mm}$. The mesh was created with 8 -node thermomechanically coupled linear Eulerian brick elements with reduced integration and hourglass control. The ABAQUS/Explicit package was used to model the welding phase (extrusion stage) of LFW.

The welding process parameters used in the model are based on previous experiments, with the oscillation frequency, amplitude of oscillation and friction pressure being $35 \mathrm{~Hz}, 3.5 \mathrm{~mm}$ and $60 \mathrm{MPa}$, respectively. The welding time is tentatively set as $1 \mathrm{~s}$ for saving computer time, while in some cases is longer. To simplify the calculation, the workpiece was assumed to have reached the equilibrium phase with a predefined temperature distribution as used by Turner et al [21]. The initial temperature of the specimen (Fig. 2) based on the simulated temperature field of the equilibrium phase $[11,12]$ is as follows:

$$
T(\mathrm{y})=25+1200 \exp \left(\frac{-\mathrm{y}^{2}}{4.5 \times 10^{-6}}\right)
$$

where $\mathrm{y}$ is the distance from the welding interface in the axial shortening direction. The highest temperature at the interface is $1225^{\circ} \mathrm{C}$ and the surrounding temperature is $25^{\circ} \mathrm{C}$. Thermal conduction within the workpiece was assumed

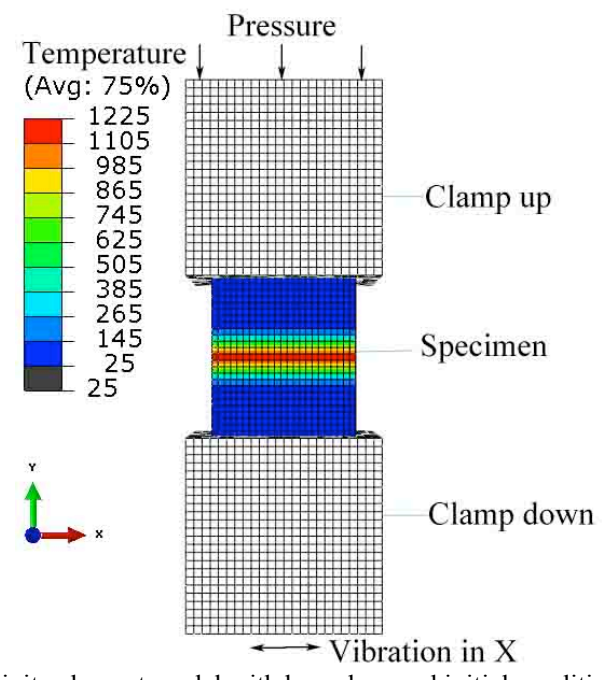

Fig. 2. Finite element model with boundary and initial conditions.

\subsection{Material properties}

The material studied in this work is the Ti6Al4V titanium alloy, the most commonly used titanium alloy in aerospace industry. The density, Young's modulus and Poisson's ratio of Ti64 are $4340 \mathrm{~kg} / \mathrm{m}^{3}, 114 \mathrm{GPa}$ and 0.34 , respectively [11]. The temperature-dependent conductivity and specific heat of Ti64 used in the model were taken from literature [11]. The dependence of material flow stress on temperature, strain and strain rate is essential for the accurate modeling of the LFW process, thus the material flow stress $(\sigma)$ was described by the Johnson-Cook plasticity model, which accounts for strain hardening, strain rate hardening and thermal softening effects [11]:

$$
\sigma=\left(A+B \varepsilon_{p}^{n}\right)\left(1+C \ln \left(\frac{\dot{\varepsilon}_{p}}{\dot{\varepsilon}_{0}}\right)\right)\left(1-\left[\frac{T-T_{r}}{T_{m}-T_{r}}\right]^{m}\right)
$$

where, $\varepsilon_{p}$ and $\dot{\varepsilon}_{p}$ are the effective plastic strain and effective plastic strain rate, respectively, $\dot{\varepsilon}_{0}$ is the reference strain rate (here is 1.0), $T_{r}$ is the reference temperature, $T_{m}$ is the melting point, $\mathrm{A}, \mathrm{B}, \mathrm{n}, \mathrm{C}$ and $\mathrm{m}$ are material dependent constants, which are $418.4 \mathrm{MPa}, 394.4 \mathrm{MPa}, 0.47,0.035$ and 1 , respectively [11].

\subsection{Micro-swing}

The welded specimens were only allowed to move in the axial direction and oscillation direction in the previous models. But during the LFW experiment, the oscillating specimen can be observed to micro-swing when studied with high-speed photography. In order to study the effect of micro-swing, this work applied different amplitudes of micro-swing (according to the observations of the highspeed photography) to the oscillatory specimen in the above model (as listed in Table 1), condition A is for the simulation model without micro-swing. Positive values for micro-swing are shown in Fig.1 (e, f) and negative values for micro-swing are shown in Fig.1 (g, h).

Table 1. Micro-swing of amplitude in the model.

\begin{tabular}{l|c|c|c|c|c|c|c}
$\frac{\text { Condition No. }}{\text { Amplitude }_{\text {of micro-swing }}}$ & $\frac{\mathbf{A}}{0^{\circ}}$ & $\frac{\mathbf{B}}{0.3^{\circ}}$ & $\frac{\mathbf{C}}{0.5^{\circ}}$ & $\frac{\mathbf{D}}{0.7^{\circ}}$ & $\frac{\mathbf{E}}{0.9^{\circ}}$ & $\frac{\mathbf{F}}{-0.7^{\circ}}$ & $\frac{\mathbf{G}}{-0.9^{\circ}}$
\end{tabular}

\section{Results} welds

3.1 Impact of micro-swing on flash formation of the

The impact of different amplitudes of micro-swing on flash formation of welds is shown in Fig. 3. During the LFW process, the viscoplastic material at the interface is continuously extruded under the simultaneous effect of oscillation, axial extrusion and micro-swing. Therefore, these three factors have a significant effect on temperature distribution at the interface. The increase of micro-swing leads to a higher temperature metal being extruded to form the flash. Therefore, the area of high-temperature zone increases with increasing the amplitude of micro-swing. Fig. 3 shows the appearance of weld and temperature field at different micro-swings. As it can be seen in Fig. 3, with increasing the amplitude of micro-swing, the flash volume is growing.

Fig. 4 shows the inner temperature fields for different micro-swings at $1 \mathrm{~s}$. The parts of less than $800^{\circ} \mathrm{C}$ were marked with the same color to clearly show temperature distribution inside the joint. Although the different amplitudes of micro-swing lead to the different weld formation, but the inner temperature field remain about the same. 


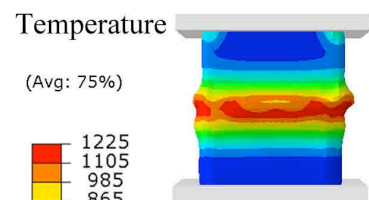

(a)

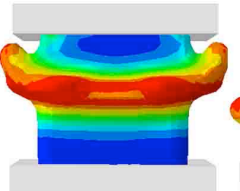

(d)

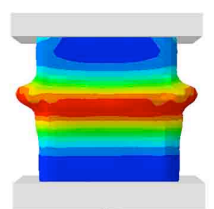

(b)

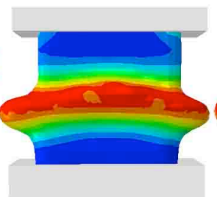

(e)

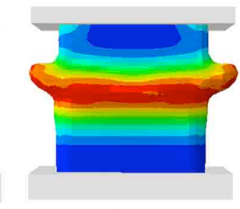

(c)

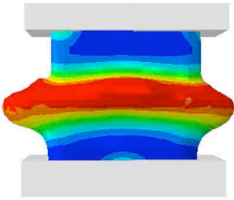

(f)
Fig. 3. Joint morphologies and temperatures for different micro-swings at $1 \mathrm{~s}$ : (a) 0 , (b) $0.3^{\circ}$, (c) $0.5^{\circ}$, (d) $0.9^{\circ}$, (e) $-0.7^{\circ}$ and (f) $-0.9^{\circ}$.

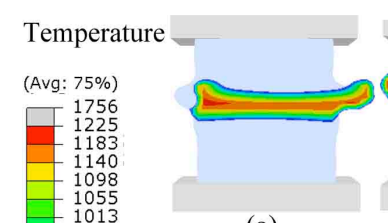

(a)

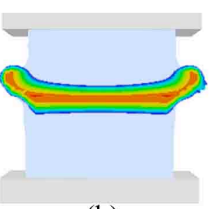

(b)

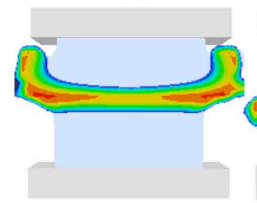

(d)

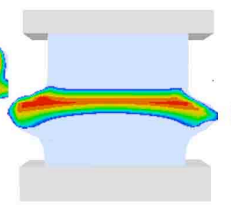

(e)

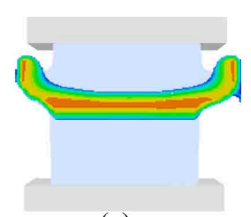

(c)

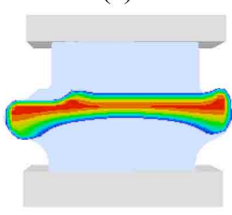

(f)
Fig. 4. Inner temperature fields for different micro-swings at 1s: (a) $0.3^{\circ}$, (b) $0.5^{\circ}$, (c) $0.7^{\circ}$, (d) $0.9^{\circ}$, (e) $-0.7^{\circ}$ and (f) $-0.9^{\circ}$.

\subsection{Impact of micro-swing on temperature field history}

Fig. 5 shows typical joint shapes and temperature distributions at different welding times. Here, the amplitudes of micro-swing are $0.7^{\circ}$ and $-0.7^{\circ}$. As time goes on into the process, high temperature material was extruded to form the flash and the high temperature region was also expanding. During the LFW process, the bend direction of flash depends on the sign of amplitude of the micro-swing. It is shown in Fig. 3 (a-d) and Fig. 5 (a-c) that the flash bends to the stationary part when the amplitude of micro-swing is positive. When the amplitude of micro-swing is negative, the bending direction of flash is shown in Fig. 3 (e,f) and Fig. 5 (d-f).

\subsection{Impact of micro-swing on axial shortening}

Fig. 7 shows the effect on axial shortening history for different amplitudes of micro-swing. There is no continuous axial shortening and there appears a short separation effect when the micro-swing is set at $0 \mathrm{o}$. In other words, when there is an absence of micro-swing causes the LFW process not to form a flash. Studying B, C, D and E cases, a certain amount of axial shortening appears at the beginning of the oscillation, then the LFW process reaches an equilibrium, and the axial shortening curve is stepped. This shows that the flash must be extruded during every oscillation cycle. Furthermore, axial shortening of welded joints increases with the amplitude of micro-swing. It is should be noted that for case E, axial shortening increases faster at $0.8 \mathrm{~s}$, but in the A-D cases axial shortening speed remains unchanged. In the $F$ and $G$ cases, sudden changes (different from the oscillation cycle) show in the curve of axial shortening history.
Temperature

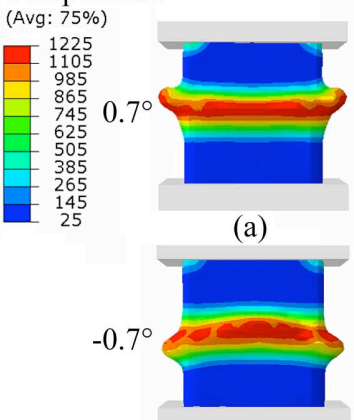

(d)

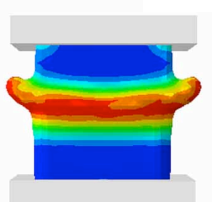

(b)

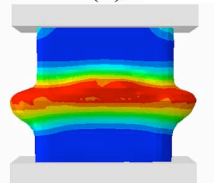

(e)

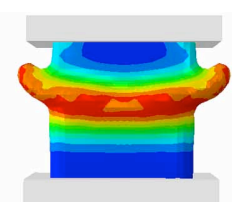

(c)

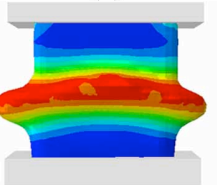

(f)
Fig. 5. Typical temperature fields at different welding times: (a) (d) $0.3 \mathrm{~s}$, (b) (e) $0.6 \mathrm{~s}$, (c) (f) $1 \mathrm{~s}$.

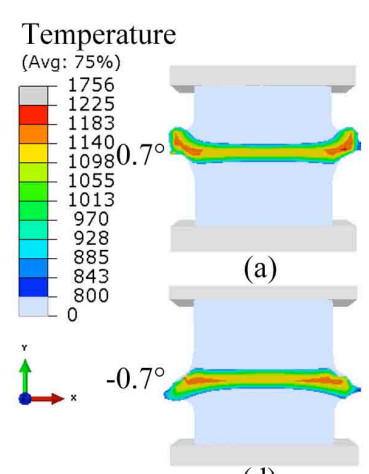

(d)

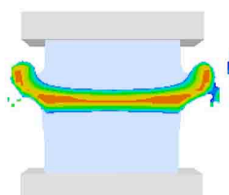

(b)

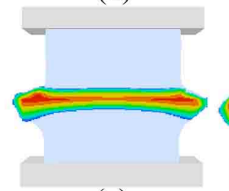

(e)

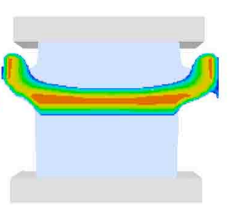

(c)

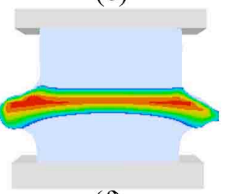

(f)
Fig. 6. Typical inner temperature fields at different welding times: (a) (d) $0.3 \mathrm{~s}$, (b) (e) $0.6 \mathrm{~s}$, (c) (f) $1 \mathrm{~s}$.

\section{Discussion}

The high speed photography showed that micro-swing is present during LFW. Possible effect of micro-swing on flash formation and axial shortening is shown in Fig. 8. When the amplitude of micro-swing is negative and the oscillating sample moves to the left as shown in Fig. 8(a), the viscoplastic metal at the welding interface is extruded into the space between the two specimens and part of the viscoplastic metal is extruded out of the welding interface to form the flash. When the oscillating sample moves to the right as shown in Fig. 8(b), the right edge of lower sample digs into the non-oscillating sample under the axial load, at the same time the viscoplastic metal between two specimens is extruded as the angle decreases. When the amplitude of micro-swing is positive and the oscillating sample moves to the left as shown in Fig. 8(c), the viscoplastic metal at the welding interface is extruded into the space between the two specimens and part of the viscoplastic metal is extruded out of the welding interface to become the flash at the right edge. The welding interface moves under the simultaneous action of oscillation, micro-swing and axial load during the LFW process. These combined effects develop a digginglike action of the viscoplastic material in the axial direction and extruding it in the direction of vibration. The more swinging the specimen, the stronger the extruding and digging effects. This results in more high-temperature metal being extruded and larger axial shortening. In future research, the extrusion behavior of interfacial metal will be explored through mechanical analysis and experiments. 


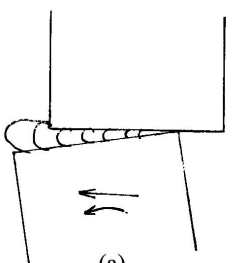

(a)

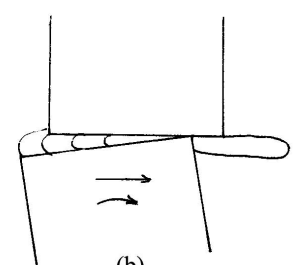

(b)

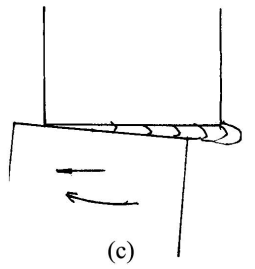

(c)
Fig. 8. Possible influence of micro-swing on flash formation and axial shortening: ( $a, b)$ amplitude of micro-swing is negative, (c) amplitude of micro-swing is positive.

When the amplitude of micro-swing is $0.9^{\circ}$, the viscoplastic metal extrudes too fast but heat is conducted away too slowly. The extrusion is faster at $0.8 \mathrm{~s}$ which is possible due to the increase in thickness of the viscoplastic metal layer of the joint resulting due to heat conduction during $0.1 \mathrm{~s}$ to $0.8 \mathrm{~s}$. When the thick viscoplastic metal layer cannot withstand the axial load, it will be extruded quickly. Similarly, for the same possible reason, when the amplitudes of micro-swing are $-0.7^{\circ}$ and $-0.9^{\circ}$, sudden changes of axial shortening will show. In future research, we will build an analytical model to analyze the heat conduction, and thus verify the above inference.

\section{Conclusions}

The developed 3D numerical model was used to simulate LFW of Ti6Al4V in different amplitudes of micro-swing, and the following conclusions can be drawn:
(1) During LFW, different levels of micro-swing could affect the flash formation, but the maximum interface temperature of the joint remained around $1200^{\circ} \mathrm{C}$ and the temperature distribution within the joint stay constant. That means heat generated through plastic deformation could maintain the welding process, and the welding process would enter the equilibrium phase.

(2) In the absence of micro-swing, the LFW process can not continuously form the flash. Micro-swing is beneficial to the extrusion of plastic metal and the formation of flash during LFW. The axial shortening of welded joints increases with micro-swing.

\section{Acknowledgements}

The authors would like to thank for financial support the Fok Ying-Tong Education Foundation for Young Teachers in the Higher Education Institutions of China (131052), the Innovation Project of Shaanxi Province Overall Plan on Science and Technology (2012HBSZS021) the 111 Project (B08040), the K.C.Wong Education Foundation, HONG KONG and the Fundamental Research Funds for the Central Universities (3102014JC02010404).

\section{References}

[1] M. Maalekian: 'Friction welding - critical assessment of literature', Sci. Technol. Weld. Join., 2007, 12, (8), 738-759.

[2] R. S. Mishra and Z. Y. Ma: 'Friction stir welding and processing', Mater. Sci. Eng. R, 2005, 50, 1-78.

[3] R. Nandan, T. DebRoy and H. K. D. H. Bhadeshia: 'Recent advances in friction-stir welding-process, weldment structure and properties', Prog. Mater. Sci., 2008, 53, (6), 980-1023.

[4] P. L. Threadgill, A. J. Leonard, H. R. Shercliff and P. J. Withers: 'Friction stir welding of aluminium alloys', Int. Mater. Rev., 2009, 54, 49-93.

[5] G. Çam: 'Friction stir welded structural materials: beyond Alalloys', Int. Mater. Rev., 2011, 56, (1), 1-48.

[6] I. Bhamji, M. Preuss, P. L. Threadgill and A. C. Addison: 'Solid state joining of metals by linear friction welding: a literature review', Mater. Sci. Technol., 2011, 27, (1), 2-12.

[7] M. E. Nunn: 'Aero engine improvements through linear friction welding', Proc. 1st Int. Conf. on 'Innovation and integration in aerospace sciences', Belfast, UK, August 2005, CEIAT, Paper 0040.

[8] A. Vairis and M. Frost: 'High frequency linear friction welding of a titanium alloy', Wear, 1998, 217, 117-131.

[9] A. Vairis and M. Frost: 'On the extrusion stage of linear friction welding of Ti 6Al 4V', Mater. Sci. Eng., 1999, 271A, 477-484.

[10] A. Vairis and M. Frost: 'Modelling the linear friction welding of titanium blocks', Mater. Sci. Eng., 2000, 292A, 8-17.

[11] W. Y. Li, T. J. Ma and J. L. Li: 'Numerical simulation of linear friction welding of titanium alloy: Effects of processing parameters', Mater. Des., 2010, 31, 1497-1507.

[12] M. Grujicic, G. Arakere, B. Pandurangan, C. F. Yen and B. A. Cheeseman: 'Process modeling of Ti-6Al-4V linear friction welding (LFW)', J. Mater. Eng. Perform., 2012, 21, (10), 20112023.

[13] L. Fratini, G. Buffa, D. Campanella and D. La Spisa: 'Investigations on the linear friction welding process through numerical simulations and experiments', Mater. Des., 2012, 40, 285-291.
[14] J. Tao, T. C. Zhang, P. T. Liu, J. Li and Y. Mang: 'Numerical computation of a linear friction welding process', Mater. Sci. Forum, 2008, 575-578, 811-815.

[15] E. Ceretti, L. Fratini, C. Giardini and D. La Spisa: 'Numerical modelling of the linear friction welding process', Int. J. Mater. Form., 2010, 3, (suppl. 1), 1015-1018.

[16] W.Y. Li, S. X. Shi, F. F. Wang, T. J. Ma, J. L. Li, D. L. Gao and A. Vairis: 'Heat reflux in flash and its effect on joint temperature history during linear friction welding of steel', Int. J. Therm. Sci., 2013, 67, 192-199.

[17] S. X. Shi, W. Y. Li, F. F. Wang, T. J. Ma and J. L. Li: '3D numerical simulation of linear friction welding of mild steel based on ABAQUS', Proc. 2nd Int. Conf. on 'The 2nd International Symposium on Computer-Aided Welding Engineering (CAWE 2012)', Jinnan, China, August 23-26, 2012.

[18] A. M. Yamileva, A. V. Yuldashev and I. Sh. Nasibullayev: 'Comparison of the parallelization efficiency of a thermo-structural problem simulated in SIMULIA Abaqus and ANSYS Mechanical', J. Eng. Sci. Technol. Rev., 2012, 5, (3), 39-43

[19] J. Sorina-Müller, M. Rettenmayr, D. Schneefeld, O. Roder and W. Fried: 'FEM simulation of the linear friction welding of titanium alloys', Comput. Mater. Sci., 2010, 48, 749-758.

[20] X. Song, M. Xie, F. Hofmann, T. S. Jun, T. Connolley, C. Reinhard, R. C. Atwood, L. Connor, M. Drakopoulos, S. Harding and A. M. Korsunsky: 'Residual stresses in linear friction welding of aluminium alloys', Mater. Des., 2013, 50, 360-369.

[21] R. Turner, J. C. Gebelin, R. M. Ward and R. C. Reed: 'Linear friction welding of Ti-6Al-4V: Modelling and validation', Acta Mater., 2011, 59, 3792-3803. 